\title{
An Eight-Week Supervised Fitness Program in Reducing Cardiovascular Risk Factors Among African-American College Students
}

\author{
Hsin-Yi Liu \\ Department of Kinesiology and Recreation Administration, North Carolina Central University, Durham, USA
}

Email address:

hliu@nccu.edu

To cite this article:

Hsin-Yi Liu. An Eight-Week Supervised Fitness Program in Reducing Cardiovascular Risk Factors Among African-American College

Students. International Journal of Sports Science and Physical Education. Vol. 3, No. 3, 2018, pp. 40-48. doi: 10.11648/j.ijsspe.20180303.12

Received: August 22, 2018; Accepted: October 31, 2018; Published: November 28, 2018

\begin{abstract}
Cardiovascular disease (CVD) is the leading cause of death of all races in the United States. African-Americans are more likely to die from CVD than whites. One hundred and forty-six African-Americans college students were recruited from a historical black college (HBCU) in North Carolina. A fitness course (PEDU 1541) was required for all students at North Carolina Central University (NCCU). An eight-week program was designed by several certified fitness instructors who teach this course at NCCU. This study was to exam the effectiveness of an 8-week exercise program in reducing CVD risk factors, including cardiovascular fitness, waist circumferences, and body fat percentage (BF\%). Students' BMI, body composition, cardiovascular fitness, muscular endurance, and trunk flexibility were assessed before and after completion of this 8-week supervised program. All the pre- and post-measurements of the 146 students were compared with a paired-t test to see if there were any significant changes. After 8 weeks of participation in this program, all measurements except their body fat composition improved $(\mathrm{p}<0.05)$. Students' diet or calorie intake was not controlled during the program in this study. Therefore, no significant change was found in their answers to the modified questionnaire of health behaviors, including alcohol, tobacco, vegetable consumptions before and after they completed this 8-week fitness course. Therefore, increase physical activity level alone may not be sufficient to reduce all risks of CVD in African-American population.
\end{abstract}

Keywords: Fitness, Aerobic, Body Composition, Muscle Endurance, African-Americans

\section{Introduction}

Cardiovascular disease (CVD) is the leading cause of death for women and man of all races and ethnicities in the United States [1]. However, racial differences in mortality rate have been well documented [2]. In 2015, 46\% of male and $48.3 \%$ of female among African Americans age 20 or older have CVD. CVD caused the deaths of 47,130 black females and 46,081 black males; $21 \%$ more than nonHispanic whites [1]. Many risk factors have been associated with CVD. Some risk factors cannot be modified, such as family history, while some are modifiable, such as physical inactivity, tobacco, diet, blood lipids, hypertension, and obesity. Studies also showed a higher prevalence of risk factors such as hypertension, diabetes mellitus, obesity, cigarette smoking, and left ventricular hypertrophy might account for the disproportionate rate of coronary heart disease mortality in African Americans [3-4]. In addition, traditional family food with high fat, sugar and sodium combine with a less active lifestyle may further pose the problem on their health for some African Americans [5].

Decades of research have shown that diet and physical activity have a major impact on CVD, diabetes, and disease risks. For example, some dietary cholesterol had significant effects on increasing total cholesterol concentrations in humans and animals [6-7]. Others showed that dietary and other lifestyle modifications, such as a reduced salt intake, increased physical activity, and weight loss can lower blood pressure and reduce incidence of hypertension and CVD [8]. Many physical activity studies also showed low cardiorespiratory fitness was a major risk factor for CVD [9-11]. Furthermore, exercise regimens not only can lower blood pressure, but also reduce the cardiovascular events and the mortality rate by $35 \%$ in patients with CVD [12-16]. However, diet pattern and lifestyle may vary 
between minority and majority population due to the cultural influence. The effects of exercise regimens on reducing different CVD risk factors among different race and ethnicities were not recorded. Therefore, additional research on the effects of exercise on African Americans is needed to refine the current public health guideline regarding physical activity.

\section{Methodology}

One hundred and fifty-six African American college students, age between 18-25, and did not have any chronic disease including type 2 diabetes, hypertension and CVD were recruited from North Carolina Central University, a historically black university. All participants registered for the required fitness class (PEDU 1541) were taught by the same instructor during the year of 2015 and 2016. Each participant was required by the Institutional Review Board (IRB) to sign a consent form before participating in the study.

\subsection{Data Collection}

Upon enrollment, baseline data of BMI, waist and hip circumference, percentage body fat, blood pressure, and cardiovascular fitness were collected from each participant. The percent body fat was estimated with a TANITA body composition analyzer. The cardiovascular fitness was measured by the recovery heart rate (bpm) after a 3-minute step exercise, one-minute sit-up, one-minute push-up and one-mile run. Their flexibility was also measure with a sit and reach test. In addition, a modified questionnaire of Youth Risk Behavior Surveillance System (CDC, 2009) for young adults (Appendix A), including the questions related to tobacco, alcohol, drug use, body weight, diet, and physical activity, was given to assess any confounding factors that may affect the result besides the exercise program. All the variables and the questionnaire were also administered at the $4^{\text {th }}$ and $8^{\text {th }}$ week after enrollment to each group. The data of the students who did not complete the 8-week exercise program and the initial, $4^{\text {th }}$, and $8^{\text {th }}$ week assessments were excluded from data analysis.

\subsection{Exercise Program}

All participants met with the instructor twice per week for 8 weeks. Each class was 50 minutes, including a 5-10 minutes warm-up and a 40-45 minutes work out sessions. During the work-out session, students were instructed to perform a combination of muscle-strengthen and Cardio exercise with supervision by the fitness instructor. The muscle-strengthen exercise include leg curl, leg press, shoulder press, biceps curl, triceps extension, chest press, abdominal, and low back muscle groups was instructed at the first day. Each participant was instructed to pick at least one exercise per large muscle group for 1 set of 15 reps for the first week, and gradually increase each week to 4 sets of 6 reps at the $8^{\text {th }}$ week. Participants were also instructed to perform one of the Cardio exercise including brisk walking, bicycling, step aerobics, and jogging for 20 minutes for the first three weeks, 25 minutes from $4^{\text {th }}$ to $6^{\text {th }}$ week, and 30 minutes for the last two weeks. A work-out log to record the exercise and time was required to be filled out by each participant after completion of the 8 -week program (Appendix B).

\subsection{Quantitative Analysis}

Changes between the pre- and post-training for anthropometric, body composition, and muscular strength, endurance and post-exercise heart rate were analyzed with a paired-t test. The significant level was assessed at $p \leq 0.05$. All analysis was conducted using IBM SPSS statistical software, version 22 .

\section{Results}

\subsection{Initial Assessment}

One hundred and forty-six (48 males and 98 females) students' data were analyzed. Their mean age was $19.24 \pm$ 1.71 years old. The average BMI of the male students was $27.58 \pm 6.60$ and female students was $28.28 \pm 7.88$ at the initial body composition assessments. Both gender groups were overweight (between 25 30) with increased risk of CVD based on the BMI classification for adults (Table 1).

Table 1. BMI categories for adults.

\begin{tabular}{lll}
\hline BMI Categories & BMI & Health Risk \\
\hline Obese & $\geq 30$ & High \\
Overweight & $\geq 25$ and $<30$ & Increased \\
Normal & $\geq 18.5$ and $<25$ & Average \\
Underweight & $<18.5$ & Increased \\
\hline
\end{tabular}

Our male students' average $\mathrm{BF} \%$ was $22.55 \pm 10.40$ considered average (between 18 24\%) but female students' average $\mathrm{BF} \%$ was $32.90 \pm 10.63$ considered obese $(>32 \%)$ based on the Percent BF Classification from American Council of Exercise (ACE) Personal Trainer Manual (2015) (Table 2). As for their averaged waist circumferences, both groups were within the low risks classification (male between 32-39in; female between 27-35in) for CVD (Table 3).

Table 2. General body fat percentage categories.

\begin{tabular}{lll}
\hline Classification & Women (\%fat) & Men (\%fat) \\
\hline Essential Fat & $10-13 \%$ & $2-5 \%$ \\
Athletes & $14-20 \%$ & $6-13 \%$ \\
Fitness & $21-24 \%$ & $14-17 \%$ \\
Average & $25-31 \%$ & $18-24 \%$ \\
Obese & $\geq 32 \%$ & $\geq 25 \%$ \\
\hline
\end{tabular}

Table 3. Initial assessment results.

\begin{tabular}{lll}
\hline Parameter & Male $(\mathbf{N}=\mathbf{4 8})$ & Female $(\mathbf{N}=\mathbf{9 8})$ \\
\hline Age & $19.10 \pm 2.28$ & $19.51 \pm 2.21$ \\
Body Weight (lb) & $190.91 \pm 50.19$ & $168.05 \pm 47.75$ \\
Height (in) & $69.76 \pm 2.96$ & $64.70 \pm 2.87$ \\
BMI (kg/m2) & $27.58 \pm 6.60$ & $28.28 \pm 7.88$ \\
BF (\%) & $22.55 \pm 10.40$ & $32.90 \pm 10.63$ \\
Waist circumference (in) & $34.38 \pm 7.35$ & $32.51 \pm 6.71$ \\
\hline
\end{tabular}




\subsection{Fitness Assessments}

There was no significant change at the end of the $4^{\text {th }}$ week of the program in any of the variable assessed. However, significant improvements were shown in: waist circumference $(2.04 \%$ decrease in males and $2.51 \%$ decrease in females), muscle endurance in sit-up $(23.87 \%$ increase in males; $32.55 \%$ increase in females), trunk flexibility $(9.39 \%$ increase in males; $12.57 \%$ in females), and post-exercise heart rate $(15.56 \%$ decrease in males; $3.48 \%$ decrease in females) at the $8^{\text {th }}$ week assessments (Table $3 \& 4$ ).

\subsection{Body Composition Assessments}

The average body weight of the male students was increased after 8 weeks of training while females' weight did not change. There was no significant change of their $\mathrm{BF} \%$ in either group, which suggested that this 8-week supervised fitness program improved their cardiovascular fitness, muscular endurance and flexibility of the participants without lowering their body weight or $\mathrm{BF} \%$ (Table 4 \& 5).

Table 4. Main parameters of male students were compared using paired $t$ test $\left(*_{p} \leq 0.05\right)$.

\begin{tabular}{llll}
\hline Parameter & Pre-Training & Post-Training & p \\
\hline Body Weight (lb) & $190.91 \pm$ & $193.02 \pm$ & $0.014^{*}$ \\
BMI (kg/m2) & 50.19 & 50.48 & \\
Waist Circumference (in) & $27.58 \pm 6.60$ & $27.87 \pm 6.62$ & $0.029^{*}$ \\
BF (\%) & $22.55 \pm 10.40$ & $22.11 \pm 9.90$ & 0.400 \\
Muscle Endurance (sit- & $32.59 \pm 13.73$ & $40.37 \pm 14.89$ & $0.001^{*}$ \\
ups/min) & $11.40 \pm 6.71$ & $12.47 \pm 6.08$ & $0.003^{*}$ \\
Trunk Flexibility (cm) & $114.84 \pm 25.63$ & $96.97 \pm 20.46$ & $0.001^{*}$ \\
\hline Post-Exercise HR (bpm) & & & \\
\hline
\end{tabular}

Table 5. Main parameters of female students were compared using paired test $(* p \leq 0.05)$.

\begin{tabular}{llll}
\hline Parameter & Pre-Training & Post-Training & p \\
\hline Body Weight (lb) & $168.13 \pm 48.11$ & $168.71 \pm 47.07$ & 0.252 \\
BMI (kg/m2) & $28.36 \pm 7.93$ & $28.41 \pm 7.70$ & 0.590 \\
Waist Circumference (in) & $32.61 \pm 6.73$ & $31.79 \pm 6.42$ & $0.001^{*}$ \\
BF (\%) & $33.01 \pm 10.76$ & $32.55 \pm 10.06$ & 0.175 \\
Muscle Endurance (sit- & $25.41 \pm 8.79$ & $33.68 \pm 9.13$ & $0.001^{*}$ \\
ups/min) & $14.00 \pm 7.28$ & $15.76 \pm 7.18$ & $0.001^{*}$ \\
Trunk Flexibility (cm) & $120.09 \pm$ & $115.91 \pm 23.107$ & $0.005^{*}$ \\
Post-Exercise HR (bpm) & 23.73 & & \\
\hline
\end{tabular}

\subsection{Health Behavior Assessments}

In addition, there was no significant change to the students' answers to the modified questionnaire of health behaviors, including alcohol, tobacco, vegetable consumptions after they completed this 8-week fitness course. Even though the students were required to read the topics related to healthy behaviors related to cardiovascular fitness and risks, this alone did not change their behaviors with healthier diet and living. Therefore, incorporating sessions focused on the knowledge of nutrition, and the alcohol and tobacco effects on cardiovascular health may be needed.

\section{Discussion}

Obesity (BMI), Waist circumference, BF\% were associated with increased risk of developing CVD, while fitness level was associated with reducing risks of CVD. Studies have shown positive effects of exercise programs on reducing some of the CVD risk factors with black and non-black youth and adolescences [17-19]. However, few studies have assessed the effects of school-based fitness programs on college students, who are at their crucial moment in developing their adulthood habits and lifestyle. A recent study had shown that, sedentary behavior of young adults University students was negatively associated and number of self-reported sedentary extracurricular activities was positively associated with waist circumferences [20]. In addition, their moderate and vigorous physical activities were negatively associated with BMI, which suggested that reducing college students' sedentary activities and increasing their vigorous physical activities level and time could reduce their waist circumference and BMI, and thus reduce their risks of developing cardiovascular diseases. Therefore, interventions to reduce sedentary behaviors and increase healthy eating is needed for this population. This study showed that an eight-week supervised fitness program, which targeted muscle strength, endurance and flexibility, was able to reduce several risk factors of CVD among African American college students. However, other factors, such as blood lipids, blood pressure were not assessed in this study, and should also be assessed in future studies.

After completing this 8-week program, students' body weight or body fat \% did not change, suggesting a longer intervention period may be needed. In addition, there was no health behavior changes with their alcohol, or vegetable consumption. Increased calorie intake during the program may have caused the no change or some increase in their body weights. Therefore, a nutrition class and dietary plan will be needed and students' calorie intake should also be assessed in the future studies.

\section{Conclusion}

This eight-week, twice per week, supervised fitness program was able to reduce their waist circumference, and improve muscular endurance, flexibility and fitness level of the African American college students, but not their body weight or BF\%. Future studies will be needed to assessed the effectiveness of this program on other CVD risk factors, such as blood lipids, and hypertension. A dietary plan should also be incorporated with the program in the future, because increase physical activity level alone may not be sufficient to reduce the risk of CVD in African-American college-aged students or in general population. 


\section{Appendix}

\section{Appendix A. Subject \#}

This survey is about health behavior. It has been developed so you can tell us what you do that may affect your health. The information you give will be used to develop better health education for young people like yourself.

DO NOT write your name on this survey. The answers you give will be kept private. No one will know what you write. Answer the questions based on what you really do.

Completing the survey is voluntary. The questions that ask about your background will be used only to describe the types of students completing this survey. The information will not be used to find out your name. No names will ever be reported. Make sure to read every question.

1. How old are you? Years old

2. What is your sex?
A Female
B Male

3. What is your race? (Select one)
A American Indian or Alaska Native
B Asian
C Black or African American
D Native Hawaiian or Other Pacific Islander
E White
F Hispanic or Latino
G Other

The next 4 questions ask about drinking alcohol. This includes drinking beer, wine, wine coolers, and liquor such as rum, gin, vodka, or whiskey. For these questions, drinking alcohol does not include drinking a few sips of wine for religious purposes.

4. During your life, on how many days have you had at least one drink of alcohol?
A 0 days
B 1 or 2 days
C 3 to 9 days
D 10 to 19 days
E 20 to 39 days
F 40 to 99 days
G 100 or more days

5. During the past 30 days, on how many days did you have at least one drink of alcohol?
A 0 days
B 1 or 2 days
C 3 to 5 days
D 6 to 9 days
E 10 to 19 days
F 20 to 29 days
G All 30 days

6. During the past 30 days, on how many days did you have 5 or more drinks of alcohol in a row, that is, within a couple of hours?
A 0 days
B 1 day
C 2 days
D 3 to 5 days
E 6 to 9 days
F 10 to 19 days
G 20 or more days

7. During the past 30 days, on how many days did you have at least one drink of alcohol on school property?
A 0 days
B 1 or 2 days
C 3 to 5 days
D 6 to 9 days
E 10 to 19 days 
F 20 to 29 days

G All 30 days

The next 5 questions ask about body weight.

8. What is your current body weight:

9. What is your current body height: $\mathrm{lb}$

10. How do you describe your weight?
A Very underweight
B Slightly underweight
C About the right weight
D Slightly overweight
E Very overweight

11. Which of the following are you trying to do about your weight?
A Lose weight
B Gain weight
C Stay the same weight
D I am not trying to do anything about my weight

12. During the past 30 days, did you exercise to lose weight or to keep from gaining weight?
A Yes
B No

13. During the past 30 days, did you eat less food, fewer calories, or foods low in fat to lose weight or to keep from gaining weight?
A Yes
B No

14. During the past 30 days, did you take any diet pills, powders, or liquids without a doctor's advice to lose weight or to keep from gaining weight? (Do not include meal replacement products such as Slim Fast.)
A Yes
B No

The next 8 questions ask about food you ate or drank during the past 7 days. Think about all the meals and snacks you had from the time you got up until you went to bed. Be sure to include food you ate at home, at school, at restaurants, or anywhere else.

15. During the past 7 days, how many times did you drink $100 \%$ fruit juices such as orange juice, apple juice, or grape juice? (Do not count punch, Kool-Aid, sports drinks, or other fruit-flavored drinks.)

A I did not drink $100 \%$ fruit juice during the past 7 days

B 1 to 3 times during the past 7 days

C 4 to 6 times during the past 7 days

D 1 time per day

E 2 times per day

F 3 times per day

G 4 or more times per day

16. During the past 7 days, how many times did you eat fruit? (Do not count fruit juice.)
A I did not eat fruit during the past 7 days
B 1 to 3 times during the past 7 days
C 4 to 6 times during the past 7 days
D 1 time per day
E 2 times per day
F 3 times per day
$\mathrm{G} 4$ or more times per day

17. During the past 7 days, how many times did you eat green salad?
A I did not eat green salad during the past 7 days
B 1 to 3 times during the past 7 days
C 4 to 6 times during the past 7 days
D 1 time per day
E 2 times per day
F 3 times per day
$\mathrm{G} 4$ or more times per day

18. During the past 7 days, how many times did you eat potatoes? (Do not count french fries, fried potatoes, or potato chips.)

A I did not eat potatoes during the past 7 days 


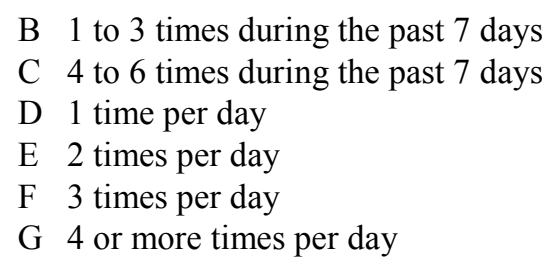

19. During the past 7 days, how many times did you eat carrots?
A I did not eat carrots during the past 7 days
B 1 to 3 times during the past 7 days
C 4 to 6 times during the past 7 days
D 1 time per day
E 2 times per day
F 3 times per day
G 4 or more times per day

20. During the past 7 days, how many times did you eat other vegetables? (Do not count green salad, potatoes, or carrots.)
A I did not eat other vegetables during the past 7 days
B 1 to 3 times during the past 7 days
C 4 to 6 times during the past 7 days
D 1 time per day
E 2 times per day
F 3 times per day
G 4 or more times per day

21. During the past 7 days, how many times did you drink a can, bottle, or glass of soda or pop, such as Coke, Pepsi, or Sprite? (Do not include diet soda or diet pop.)
A I did not drink soda or pop during the past 7 days
B 1 to 3 times during the past 7 days
C 4 to 6 times during the past 7 days
D 1 time per day
E 2 times per day
F 3 times per day
G 4 or more times per day

22. During the past 7 days, how many glasses of milk did you drink? (Include the milk you drank in a glass or cup, from a carton, or with cereal. Count the half pint of milk served at school as equal to one glass.)
A I did not drink milk during the past 7 days
B 1 to 3 glasses during the past 7 days
C 4 to 6 glasses during the past 7 days
D 1 glass per day
E 2 glasses per day
F 3 glasses per day
G 4 or more glasses per day

The next 5 questions ask about physical activity.

23. On how many of the past 7 days did you exercise or participate in sports activities for at least 20 minutes that made you sweat and breathe hard, such as basketball, jogging, swimming laps, tennis, fast bicycling, or similar aerobic activities?
A 0 days
B 1 day
C 2 days
D 3 days
E 4 days
F 5 days
G 6 days
H 7 days

24. On how many of the past 7 days did you do stretching exercises, such as toe touching, knee bending, or leg stretching?
A 0 days
B 1 day
C 2 days
D 3 days
E 4 days 

F 5 days
G 6 days
H 7 days

25. On how many of the past 7 days did you walk or bicycle for at least 30 minutes at a time? (Include walking or bicycling to or from class or work.)
A 0 days
B 1 day
C 2 days
D 3 days
E 4 days
F 5 days
G 6 days
H 7 days

26. On how many of the past 7 days did you do exercises to strengthen or tone your muscles, such as push-ups, sit-ups, or weight lifting?
A 0 days
B 1 day
C 2 days
D 3 days
E 4 days
F 5 days
G 6 days
H 7 days

27. During this school year, on how many college sports teams (intramural or extramural) did you participate?
A. 0 teams
B. 1 team
C. 2 teams
D. 3 or more teams

The next 11 questions ask about tobacco use.

28. Have you ever tried cigarette smoking, even one or two puffs?
A Yes
B No

29. Does either of your parents smoke cigarette?
A Yes
B No

30. How old were you when you smoked a whole cigarette for the first time?
A I have never smoked a whole cigarette
B 8 years old or younger
C 9 or 10 years old
D 11 or 12 years old
E 13 or 14 years old
F 15 or 16 years old
G 17 years old or older

31. During the past 30 days, on how many days did you smoke cigarettes?
A 0 days
B 1 or 2 days
C 3 to 5 days
D 6 to 9 days
E 10 to 19 days
F 20 to 29 days
G All 30 days

32. During the past 30 days, on the days you smoked, how many cigarettes did you smoke per day?
A I did not smoke cigarettes during the past 30 days
B Less than 1 cigarette per day
C 1 cigarette per day
D 2 to 5 cigarettes per day
E 6 to 10 cigarettes per day 
F 11 to 20 cigarettes per day

$\mathrm{G}$ More than 20 cigarettes per day

33. During the past 30 days, on how many days did you use chewing tobacco, snuff, or dip, such as Redman, Levi Garrett, Beechnut, Skoal, Skoal Bandits, or Copenhagen?
A 0 days
B 1 or 2 days
C 3 to 5 days
D 6 to 9 days
E 10 to 19 days
F 20 to 29 days
G All 30 days

34. During the past 30 days, on how many days did you use chewing tobacco, snuff, or dip on school property?
a) 0 days
b) 1 or 2 days
c) 3 to 5 days
d) 6 to 9 days
e) 10 to 19 days
f) 20 to 29 days
g) All 30 days

\section{Appendix B. Work Out Log Sheet}

Name:

Turn in Work out log sheets during post testing week for 5 points.

You should:

1. Determine your Goals (Muscle strength, Endurance, or Size)

2. Select Exercises (At least one exercise per large muscle group)

3. Arrange Exercises (do the hardest exercises first, squats, bench press then smaller muscles)

4. Decide on training frequency (how many days per week)

5. Determine how much weight/number of reps/number of sets

\begin{tabular}{|c|c|c|c|c|c|c|c|c|}
\hline Date & $\begin{array}{l}1 \\
\text { week }\end{array}$ & $\begin{array}{l} \\
\text { week }\end{array}$ & $\begin{array}{l}3 \\
\text { week }\end{array}$ & $\begin{array}{l}4 \\
\text { week }\end{array}$ & $\begin{array}{l}5 \\
\text { week } \\
\end{array}$ & $\begin{array}{l}6 \\
\text { week }\end{array}$ & $\begin{array}{l}7 \\
\text { week }\end{array}$ & $\begin{array}{l}8 \\
\text { week }\end{array}$ \\
\hline Exercise $^{* * * * * * *}$ & $\begin{array}{l}\mathrm{x} 15 \\
\text { Sets/reps }\end{array}$ & $\begin{array}{l}2 \times 15 \\
\text { Sets/reps }\end{array}$ & $\begin{array}{l}\mathbf{2 \times 2 0} \\
\text { Sets/Reps }\end{array}$ & $\begin{array}{l}2 \times 8 \\
\text { Sets/Reps }\end{array}$ & $\begin{array}{l}\mathbf{3 x 8} \\
\text { Sets/reps }\end{array}$ & $\begin{array}{l}\mathbf{3 x 8} \\
\text { Sets/reps }\end{array}$ & $\begin{array}{l}4 \times 6 \\
\text { Sets/reps }\end{array}$ & $\begin{array}{l}466 \\
\text { Sets/reps }\end{array}$ \\
\hline
\end{tabular}

Leg

Leg press

Leg ext

Leg curl

Arm

Shoulder press

Biceps curl

Triceps ext

Core/Trunk

Chest Press

Lat. pull-down

Abdominals

Low back

CARDIO

20

Other exercises:

$20 \quad 20 \quad 25$

Min $\quad$ Min $\quad$ Min
Min Min
25

Min
30

Min
30

Min

\section{References}

[1] American Heart Association. Heart Disease and Stroke Statistics - 2016 Update. Dallas, Texas: American Heart Association, 2016.
[2] Mensah et al. An Overview of Cardiovascular Disease Burden in the United States. Health Affairs. 2007; 26(1): 38-48.

[3] Potts JL, Thomas J. Traditionally coronary risk factors for African Americans. Am J Med Sci. 1999; 317(3): 189-92.

[4] Smith SC Jr. Multiple risk factors for cardiovascular disease and diabetes mellitus. Am J Med. 2007; 120: S3-S11. 
[5] Carson JA, Michalsky L, Latson B, Banks K, Tong L, Gimpel N, Lee JJ, Dehaven MJ. The cardiovascular health of urban African Americans: diet-related results from the Genes, Nutrition, Exercise, Wellness, and Spiritual Growth (GoodNEWS) trial. J Acad Nutr Diet. 2012; 112(11): 1852-8.

[6] John D. Griffin, M. S. and Alice H. Lichtenstein, D. Sc. Dietary Cholesterol and Plasma Lipoprotein Profiles: Randomized-Controlled Trials. Curr Nutr Rep. 2013; 2(4): 274-282.

[7] Robert JN, Arthur FS, Mark CK, Lori KH, D. Mark H, and Ernst JS. Effect of Dietary Fat Saturation and Cholesterol on LDL Composition and Metabolism. Arterioscler Thromb Vasc Biol. 1990; 10: 119-28.

[8] Lawrence JA. Lifestyle Modification as a Means to Prevent and Treat High Blood Pressure. JASN. 2003; 14 (suppl 2): S99-S102.

[9] Morris, J. N., Clayton, D. G., Everitt, M. G., Semmence, A. M., \& Burgess, E. H. Exercise in leisure time: Coronary attack and death rates. British Heart Journal. 1990; 63: 325-334.

[10] Paffenbarger RS Jr. Contributions of epidemiology to exercise science and cardiovascular health. Medicine and Science in Sports and Exercise. 1988; 20: 426-438.

[11] Paffenbarger RS Jr, Hyde RT, Wing AL, Lee I-M, Jung DL, Kampert JB. The association of changes in physical activity level and other lifestyle characteristics with mortality among men. New England Journal of Medicine. 1993; 328: 538-545.

[12] Douglas D, Caroline R, Elizabeth AJ. Physical Activity and Exercise for Secondary Prevention among Patients with Cardiovascular Disease. Curr Cardiovasc Risk Rep. 2013; 7(6): 10.1007/s12170-013-0354-5.

[13] Nocon, M., Hiemann, T., Muller-Riemenschneider, F., Thalau, F., Roll, S. and Willich, S. N. Association of physical activity with all-cause and cardiovascular mortality: a systematic review and meta-analysis. Eur. J. Cardiovasc. Prev. Rehabil. $2008 ; 15,239-246$.

[14] Paffenbarger RS Jr, Hyde RT, Wing AL, Steinmetz CH. A natural history of athleticism and cardiovascular health. JAMA. 1984; 252: 491-495.

[15] Paffenbarger RS Jr, Jung DL, Leung RW, Hyde RT. Physical activity and hypertension: an epidemiological view. Annals of Medicine. 1991; 23: 319-327.

[16] Riccardo DG, Simona Ci, Elena C, Marwan EG, Giulio M. Cognitive-Behavioral Strategies to Increase the Adherence to Exercise in the Management of Obesity. J Obes. 2011; 2011: 348293.

[17] Jamerson T, Sylvester R, Jiang Q, Corriveau N, DuRusselWeston J, Kline-Rogers E, Jackson EA, Eagle KA. Differences in Students Participating in a School-Based Health Promotion Program. Am J Health Promot. 2017; 31(4): 318-324.

[18] Berry DC, McMurray RG, Schwartz TA, Adatorwovor R. Benefits for African American and white low-income 7-10year-old children and their parents taught together in a community-based weight management program in the rural southeastern United States. BMC Public Health. 2018; 18(1): 1107.

[19] Harrell JS, McMurray RG, Bangdiwala SI, Frauman AC, Gansky SA, Bradley CB. Effects of a school-based intervention to reduce cardiovascular disease risk factors in elementary-school children: the Cardiovascular Health in Children (CHIC) study. J Pediatr. 1996; 128(6): 797-805.

[20] Peterson NE, Sirard JR, Kulbok PA, DeBoer MD, Erickson JM. Sedentary behavior and physical activity of young adult university students. Res Nurs Health. 2018; 41(1): 30-38. 